\title{
Pesticidas no perfil de solos em áreas de plantação de cebolas em Ituporanga, SC
}

\author{
$\overline{\text { Adilson Pinheiro }^{1} \text {, João C. S. Moraes }}{ }^{2} \&$ Marcos R. da Silva ${ }^{1}$
}

\begin{abstract}
RESUMO
O bjetivou-se com este trabalho avaliar as concentrações de resíduos de pesticidas no perfil do solo sob cultura de cebola, no município de Ituporanga, em Santa Catarina. Coletas de amostras foram realizadas nas profundidades de 0 a 20, 40 a 60 e 80 a $100 \mathrm{~cm}$, em três áreas com plantação de cebola. Nestas áreas são realizadas rotações de cultura, abrangendo o milho, cebola, batata, feijão e beterraba. Três séries de amostragens foram realizadas nos períodos de 2006 e 2007, sendo coletadas, em um ano, amostras simples e, no outro, uma amostra composta. Para cada amostra foram determinadas as concentrações dos herbicidas: 2,4-D, alacloro, atrazina, diurom, dos fungicidas: azoxistrobina, metalaxil, metconazol, tebuconazol e do inseticida: lambda-cialotrina. Os resultados mostram que os ingredientes ativos considerados foram detectados nas três profundidades. Os fungicidas foram detectados com maior frequência na camada superficial. As concentrações variaram entre as áreas e entre as profundidades, de forma descontínua. 0 comportamento de distribuição das concentrações foi similar para todos os pesticidas. Supõe-se que a existência de caminhos preferenciais no perfil do solo seja 0 fator responsável pela ocorrência de concentrações elevadas em profundidade, de forma irregular.
\end{abstract}

Palavras-chave: lixiviação, resíduos de pesticidas no solo, herbicida, inseticida, fungicida

\section{Pesticides in the soil profile in planting areas of onions in Ituporanga, SC}

\begin{abstract}
This study aims to evaluate the pesticide concentrations in the soil profile under cultivation of onion in the municipality of Ituporanga in Santa Catarina. Samples of soil were collected, at depths of 0-20, 40-60 and $80-100 \mathrm{~cm}$ in three areas. In these the maize, onion, potatoes, beans, and beets are used in crop rotation. Three sets of samples were collected in the period 2006 and 2007. In the first year single samples were collected and in the other year, a composite sample. For each sample, the concentrations of herbicide 2,4-D, alachlor, atrazine, diuron, of fungicides: azoxystrobin, metalaxyl, metconazole, tebuconazole and of insecticides: lambda-cyhalothrin were determined. The results show that the active ingredients were detected in three depths. The fungicides were detected more frequently in the surface layer. Those, in turn, were detected more frequently in the deeper layer. The concentrations varied among the areas and depths, occurring in discontinuous form. The behavior of the distribution of concentrations was similar for all pesticides. It is supposed that the existence of preferential paths in the soil profile is the responsible factor for the irregular occurrence of high concentrations in depth.
\end{abstract}

Key words: leaching, pesticides residues in soil, groundwater degradation potential

\footnotetext{
${ }^{1}$ U niversidade Regional de Blumenau, Rua São Paulo 3250, CEP 89030-000, Blumenau, SC. Fone: (47) 3221-6078. E-mail: pinheiro@furb.br, rivail@furb.br

${ }^{2}$ FAE Blumenau, Rua Santo Antonio s/n, CEP 89010-110, Blumenau, SC. Fone: (47) 32216077. E-mail: mentorjc@terra.com.br
} 


\section{INTRODUÇÃO}

O uso intensivo de pesticidas no Brasil iniciou-se com a revolução verde, nos anos 60. Em 2008 foram comercializados cerca de $30 \%$ de inseticidas, $21 \%$ de fungicidas e $48 \%$ de herbicidas (SINDAG, 2009). As culturas de soja, cana-de-açúcar, algodão e milho, respondem pela maior participação na venda de pesticidas. Existem no mercado cerca de 1500 diferentes ingredientes ativos com ação química e utilizados na produção de diversas formulações aplicadas na agricultura.

Os pesticidas geram problemas à saúde pública (Wade et al., 1998; Ramos et al., 2002; Peres \& Moreira 2007; Gibson \& Koifman, 2008) e ao meio ambiente (Bergstrom, 2004, Hartemink, 2008). Resíduos de pesticidas são encontrados em corpos de águas superficiais (Molozzi et al., 2006) e subterrâneos (Blanchard \& Lerch, 2000; Lapworth et al., 2006; Sakaliene et al., 2007), no solo (Vieira et al., 2009), no ar (Kromer et al., 2004, Yao et al., 2008) e em alimentos (Siqueira \& Kruse, 2008; Cantarutti et al., 2009). A dinâmica dos pesticidas, segundo Gustafson (1989) indica sua periculosidade aos sistemas ambientais, em que dois fenômenos são os principais indicadores: a mobilidade e a persistência no meio ambiente, ou seja, a capacidade de sorção à fase sólida e o tempo de meia vida da molécula; ressalta-se que diferentes índices são utilizados para representá-los, sendo o índice GUS (Groundwater Ubiquity Score) um dos mais utilizados. Moléculas com valores de GUS inferiores a 1,8 apresentam baixo potencial de lixiviação enquanto moléculas com valores superiores a 2,8 apontam potencial de lixiviação elevado e moléculas com valores entre 1,8 e 2,8 pertencem a um grupo de transição.

Por outro lado, estudos realizados com colunas de solo em laboratório (Matallo et al., 2005; Al-Rajab et al., 2008; Ruggieri et al., 2008; Vasconcelos et al., 2009) ou em parcelas experimentais (Correia \& Langenbach, 2006, Delgado-Moreno et al., 2007) indicam que os pesticidas apresentam evolução espacial e temporal das concentrações descrita por decréscimo em profundidade e redução da massa disponível com o tempo, com condições não alteradas ao longo do tempo; no entanto, em condições operacionais de campo agrícola os fatores ambientais são mais dinâmicos e há heterogeneidade espacial e temporal, que influenciam a distribuição espacial, seja na direção vertical quanto na direção horizontal do solo e no tempo, ocorrendo dissipação do produto ou por degradação, evaporação ou absorção pelos organismos.

Visa-se, neste trabalho, determinar a distribuição de pesticidas no perfil do solo de uma área agrícola cultivada com plantio convencional e com rotação de culturas. Nas áreas são realizados dois cultivos anuais, em que a cebola é a principal cultura plantada na região e onde foram feitas as amostragens; a área é cultivada pela família do produtor rural há cerca de 4 décadas. O solo é classificado como Ca 78 - Cambissolo Húmico proeminente, textura argilosa e média, fase floresta subtropical perenifólia e relevo suave ondulado (EMBRAPA, 1999). Foram determinadas concentrações de resíduos dos pesticidas usados na área, em três séries de amostragens, no período de 2006 e 2007, em três profundidades, 0-20, 40-60 e 80-100 cm. Para cada amostra foram determinados os resíduos dos herbicidas: 2,4-D, alacloro, atrazina e diurom, recomendados para a cultura do milho, dos fungicidas azoxistrobina, metalaxil, metconazol e tebuconazol e do inseticida lambda-cialotrina, recomendados para as culturas da cebola, batata e beterraba.

\section{MATERIAL E MÉTODOS}

A metodologia compreendeu a coleta de amostra de solo Ca78 - Cambissolo Húmico, com histórico de plantio convencional, sendo dois cultivos anuais, principalmente de cebola com rotação de milho, batata, feijão e beterraba. e em três áreas localizadas no município de Ituporanga, região do Alto Vale do Itajaí, Estado de Santa Catarina e a determinação das concentrações dos pesticidas na matriz do solo; por ocasião das coletas as áreas estavam com cultivo de cebola.

As amostras de solo foram coletadas com um trado holandês, de $4,5 \mathrm{~cm}$ de diâmetro e $16 \mathrm{~cm}$ de comprimento, nas profundidades de 0 a 20, 40 a 60 e 80 a $100 \mathrm{~cm}$. Os pontos de amostragem foram definidos aleatoriamente de modo a se obter amostras representativas e dois procedimentos de amostragem foram adotados, em que o primeiro consistiu na coleta de uma amostra simples obtida em um único ponto de cada área; as amostragens foram realizadas nos dias 12/10/2006 e 10/12/2006 e o segundo compreendeu a formação de uma amostra composta obtida pela mistura da coleta de solo de três pontos de amostragem em cada área, para obtenção de uma amostra mais homogênea, procedimento realizado no dia 12/10/2007.

As amostras de solo foram acondicionadas em sacos plásticos e mantidas refrigeradas a $4^{\circ} \mathrm{C}$, durante $48 \mathrm{~h}$, para posterior extração e análise por meio de cromatografia em fase líquida de alta eficiência (CLAE). Foram determinadas as concentrações das moléculas dos pesticidas apresentadas na Tabela 1.

A vidraria utilizada no processo de extração foi toda descontaminada com bastante água e detergente e com solução alcoólica de hidróxido de potássio $0,5 \mathrm{~N}$. As vidrarias não volumétricas foram aquecidas a $400^{\circ} \mathrm{C}$ em forno mufla, durante $4 \mathrm{~h}$; a vidraria volumétrica foi devidamente calibrada; os solventes utilizados foram o metanol, o diclorometano e a acetonitrila, grau HPLC, obtidos junto a JT Baker; o sulfato de sódio utilizado foi grau PA da MERCK, ativado a $400^{\circ} \mathrm{C}$ por $1 \mathrm{~h}$, em forno mufla.

Utilizaram-se, para verificação da eficiência de extração e acompanhamento do método, o surrogate decaclorobifenil e o padrão interno 2,4,5,6 - tetracloro-m -xileno. Ambas as moléculas foram adquiridas junto à empresa SUPELCO e, na calibração do cromatógrafo gasoso, foram utilizadas as moléculas-padrão 2,4-D, alacloro, atrazina, diurom, azoxistrobina, metalaxil, metconazol, tebuconazol e lambdacialotrina, obtidas individualmente, na SUPELCO. Todas as amostras de solo estudadas foram liofilizadas antes do processo de extração e a água utilizada foi purificada no sistema Milli-Q com resistividade de $18 \mathrm{MW}$.

Na extração seguiu-se o procedimento seguinte: em $50 \mathrm{~g}$ de solo liofilizado foram adicionados $150 \mu \mathrm{L}$ da mistura surrogate em frascos Erlenmeyer de $125 \mathrm{~mL}$ de capacidade, fechados com folhas de alumínio e em seguida agitadas durante 20 a 30 min em temperatura ambiente e agitação moderada. Adicionou- 
Tabela 1. Características dos ingredientes ativos anal isados

\begin{tabular}{|c|c|c|c|c|}
\hline Ingrediente ativo & Nomenclatura & $\mathrm{DT}_{50}(\mathrm{~d})$ & Koc $\left(\mathrm{mL} \mathrm{g}^{-1}\right)$ & GUS \\
\hline \multicolumn{5}{|c|}{ I - Herbicidas } \\
\hline 2,4-D & (2,4-dichlorophenoxy) acetic acid & $4.6-17.2$ & 56 & 2,25 \\
\hline Alacloro & 2-chloro-2,6-diethyl-N-methoxymethyl acetanilide & $4-24$ & $102-150$ & 2,19 \\
\hline Atrazina & 2-chloro-4-ethylamino-6-isopropylamino-s-triazine & $12-108$ & 100 & 3,75 \\
\hline Diurom & 3-(3,4-diclorofenil)-1,1-dimetiluréia & $56-231$ & $29-902$ & 1,83 \\
\hline \multicolumn{5}{|c|}{ II - Fungicidas } \\
\hline Azoxistrobina & methyl (E)-2-\{2-[6-(2-cyanophenoxy)pyrimidin-4-yloxy]-phenyl\}-3-methoxyacrylate & $2,6-39$ & 423 & 2,53 \\
\hline Metconazol & 5-(4-chlorobenzyl)-2,2-dimethyl-1-(1H-1,2,4-triazol-1-ylmethyl)-cyclopentanol & $33-845$ & $1019-1592$ & 1,83 \\
\hline Metalaxil & methyl N-(2-methoxyacetyl)-N-2,6-xylyl-D-alanina & 46 & 165 & 2,89 \\
\hline Tebuconazol & ((RS)-1-p-chlorophenyl-4,4-dimethyl-3-(1H-1,2,4-triazol-1-ylmethyl)pentan-3-ol & 62 & $803-1251$ & 1,45 \\
\hline \multicolumn{5}{|c|}{ III - Ins eticidas } \\
\hline Lambda-cialotrina & $\begin{array}{l}\lambda \text {-cyano-3-phenoxybenzyl-3-(2-chloro-3,3,3-trifluoroprop-1-enyl)-2,2-dimethyl } \\
\text { cyclopropanecarboxylate }\end{array}$ & $6-40$ & 157000 & $-1,67$ \\
\hline
\end{tabular}

se metanol o suficiente para cobrir a amostra, levando-se para agitar novamente por $10 \mathrm{~min}$; filtrou-se a amostra em papel filtro azul, recolhendo o filtrado; repetiu-se o processo de extração e se ajustou o volume final para $5 \mathrm{~mL}$ em balão volumétrico; reduziu-se o filtrado até quase secura em evaporador rotatório, restando $\pm 1 \mathrm{~mL}$ da amostra; transferiuse quantitativamente o resíduo para um funil de separação e se o diluiu com água purificada no sistema Milli-Q; repetindo-se o processo de extração mais duas vezes, com diclorometano; passou-se então o diclorometano em uma coluna de sulfato de sódio anidro, recolhendo-o em balão volumétrico e se evaporou até a secura, ressuspendendo o resíduo em $0,5 \mathrm{~mL}$ de acetonitrila/água (3:7); enfim, filtrou-se o resíduo em filtro 0,45 $\mu \mathrm{m}$, completando o volume em-balão volumétrico de $1 \mathrm{~mL}$, que foi acondicionado para análise.

Realizou-se a quantificação dos pesticidas em um cromatógrafo líquido Varian ${ }^{\circledR}$ ProStar 230, com detector UV-Vis ProStar 310 e comprimento de onda de trabalho de $214 \mathrm{~nm}$. Foi empregada coluna C18 (250 x 4,6 mm, filme $5 \mathrm{~mm})$. Para a fase móvel utilizaram-se, inicialmente, álcool metílico $0 \%$, acetonitrila $30 \%$ e $\mathrm{H}_{2} \mathrm{O} 70 \%$, equilibrando os solventes no final; o fluxo dos solventes na coluna foi de $1 \mathrm{~mL} \mathrm{~min}^{-1}$, o tempo de corrida foi de 30 min, com pressão máxima de 400 atm e pressão mínima de 6 atm e o volume do extrato injetado foi de $1 \mathrm{~mL}$; determinou-se um pesticida através de procedimento analítico de multirresíduo.

\section{RESULTADOS E DISCUSSÃO}

Nas três campanhas de amostragem foram coletadas 3 amostras, perfazendo o total de 9 amostras em cada profundidade considerada. Na Tabela 2 são apresentadas as frequências de detecção dos pesticidas em cada profundidade, as quais variaram entre 0 e $77,8 \%$. O herbicida alacloro não foi detectado na profundidade de 40 a $60 \mathrm{~cm}$; entretanto, foi detectado nas camadas superior e inferior a esta, com $11,1 \%$ de frequência em ambas.

O alacloro é estimado como tendo baixa lixiviação em solos contendo altos teores de argila e baixos teores de areia; sua mobilidade e persistência são fortemente influenciadas pelo tipo de solo e condições ambientais, tais como temperatura,
Tabela 2. Frequência (\%) de detecção dos ingredientes ativos

\begin{tabular}{lccc}
\hline \multirow{2}{*}{ Ingrediente ativo } & \multicolumn{3}{c}{ Profundidade (cm) } \\
\cline { 2 - 4 } & $\mathbf{0}$ a $\mathbf{~ 2 0}$ & $\mathbf{4 0}$ a $\mathbf{6 0}$ & $\mathbf{8 0}$ a $\mathbf{~ 1 0 0}$ \\
2,4-D & 33,3 & 22,2 & 55,6 \\
Alacloro & 11,1 & 0,0 & 11,1 \\
Atrazina & 44,4 & 44,4 & 55,6 \\
Diurom & 33,3 & 55,6 & 22,2 \\
Azoxistrobina & 44,4 & 33,3 & 22,2 \\
Metconazol & 55,6 & 33,3 & 11,1 \\
Methalaxyl & 55,6 & 66,7 & 55,6 \\
Tebuconazol & 77,8 & 22,2 & 55,6 \\
Lambda-cialotrina & 66,7 & 55,6 & 55,6 \\
\hline
\end{tabular}

atividade microbiana, níveis de oxigênio e pH (Johnson \& Sims, 1993; Kaur et al., 2002; Sakaliene et al., 2007). O fungicida tebuconazol apresentou a maior frequência em detecção na profundidade de 0 a $20 \mathrm{~cm}$. Considerando-se o conjunto das três profundidades, as maiores frequências de detecção foram determinadas ao fungicida metalaxil e ao inseticida lambdacialotrina, enquanto os fungicidas foram mais frequentemente detectados que os herbicidas, cuja diferença foi de aproximadamente $37 \%$ (Tabela 2) devido, sem dúvida, ao tempo passado desde a aplicação das culturas citadas, em que os herbicidas só têm registro para serem aplicados no milho e os fungicidas e o inseticida na cebola. Os fungicidas são aplicados com maior frequência podendo não ter tido tempo suficiente de dissipar por degradação ou mobilidade.

Em geral, no perfil do solo os ingredientes ativos foram encontrados sobretudo na profundidade de 0 a $20 \mathrm{~cm}$, com frequência média de $47 \%$ das amostras enquanto nas camadas mais profundas a frequência foi da ordem de $37,5 \%$. Os herbicidas foram detectados com maior frequência $(\sim 18 \%)$ na profundidade de 80 a $100 \mathrm{~cm}$ que nas profundidades de 0 a 20 e 40 a $60 \mathrm{~cm}$, mostrando mobilidade com o tempo de aplicação. Por outro lado, os fungicidas foram cerca de $50 \%$ mais detectados na profundidade de 0 a $20 \mathrm{~cm}$ que nas outras duas profundidades apresentando, portanto, maior adsorção na região de superfície devido, provavelmente, ao tempo de aplicação (possuem registro para serem utilizados em cultivo de cebola) e se sabe que os fungicidas são mais persitentes no solo que os herbicidas. Alguns pesquisadores também encontraram dois fungicidas: o metalaxil e o clorotalonil, em 
maior quantidade na camada de aproximadamente $10 \mathrm{~cm}$ atribuindo-se, a isto, o alto teor de carbono orgânico e o revolvimento do solo da superfície (Wu et al., 2002).

Através da frequência de detecção nota-se que os pesticidas alcançam a profundidade de 80 a $100 \mathrm{~cm}$, podendo representar potencial de contaminação das águas subterrâneas. Analisando os pesticidas em termos do potencial de lixiviação através do índice GUS (Tabela 1), esperava-se o herbicida atrazina e o fungicida metalaxil com maior frequência nas camadas inferiores (40 a $100 \mathrm{~cm}$ ) que os outros produtos, de menor índice GUS, como o fungicida tebuconazol e o inseticida lambda-cialotrina; entretanto, ambos ocorreram na mesma frequência que o atrazina devido, sem dúvida, às propriedades do solo, do tempo passado da aplicação ou da prática agrícola utilizada. Esses resultados mostram a importância do monitoramento dos solos e das águas de todas as substâncias utilizadas uma vez que, com o tempo, frequência de utilização e quantidade utilizada podem levar a contaminações do ambiente.

$\mathrm{Na}$ Tabela 3 se acham as concentrações dos pesticidas determinados nas amostras, sejam elas simples (10/2006 e 12/ 2006) ou compostas (10/2007), em que dois aspectos devem ser observados: primeiro, os valores das concentrações foram bastante variáveis e o maior valor foi determinado no herbicida 2,4-D, na coleta de 12/2006 (847,5 $\left.\mathrm{ng} \mathrm{g}^{-1}\right)$ na profundidade de 0 a $20 \mathrm{~cm}$, na área amostral A3; a menor concentração detectada foi do herbicida atrazina, na área $\mathrm{A} 1$, na profundidade de $80 \mathrm{a}$ $100 \mathrm{~cm}$, cujo valor foi de $2,1 \mathrm{ng} \mathrm{g}^{-1}$ porém tanto o herbicida 2,4-
D quanto a atrazina, tal como os demais pesticidas, apresentaram valores de concentrações variáveis na mesma profundidade e entre as áreas, significando que o procedimento amostral não influenciou o resultado; já o segundo aspecto está relacionado à não continuidade das concentrações, sejam elas entre as áreas ou entre as profundidades, fato constatado para todos os pesticidas analisados. As práticas agrícolas desenvolvidas nas três áreas são idênticas, sendo adotado o plantio convencional com reviramento do solo, o que promove a desestruturação da camada superficial facilitando a infiltração e percolação da água no perfil do solo; o escoamento da água promove o transporte dos pesticidas.

Os escoamentos, no entanto, não ocorrem de forma homogênea e os solos são heterogêneos, envolvendo diferentes escalas espaciais que geram variabilidade nas propriedades físicas (Schaffrath et al., 2008). A existência de macroporos produz caminhos preferenciais com relação ao sistema restante, produzindo um tempo de resposta bem menor que aquele através da matriz do solo, interconectando-se rapidamente às camadas do solo afetadas. Eles podem ser formados pela fauna, pelas raízes das plantas, fissuras e rachaduras e condutos naturais dentro do solo (Malone et al., 2004) e isto pode explicar a ocorrência de valores diferentes das concentrações dos pesticidas nas áreas e profundidades amostradas. $\mathrm{O}$ entendimento da variabilidade das propriedades e processos do solo como função da profundidade, é importante para uma predição exata da dissipação do pesticida (Papiernik et al., 2006).

Tabela 3. Concentrações $\left(\mathrm{ng} \mathrm{g}^{-1}\right)$ nas diferentes áreas (A1, A2, e A3) e profundidades

\begin{tabular}{|c|c|c|c|c|c|c|c|c|c|c|}
\hline \multirow{3}{*}{ Ingrediente Ativo } & \multirow{3}{*}{ Data } & \multicolumn{9}{|c|}{ Profundidade $(\mathrm{cm})$} \\
\hline & & \multicolumn{3}{|c|}{0 a 20} & \multicolumn{3}{|c|}{40 a 60} & \multicolumn{3}{|c|}{80 a 100} \\
\hline & & A1 & A2 & A3 & A1 & A2 & A3 & A1 & A2 & A3 \\
\hline $2,4-D$ & $\begin{array}{l}10 / 2006 \\
12 / 2006 \\
10 / 2007\end{array}$ & $\begin{array}{l}8,8 \\
8,1 \\
\text { nd }\end{array}$ & $\begin{array}{l}\text { nd } \\
\text { nd } \\
\text { nd }\end{array}$ & $\begin{array}{c}\text { nd } \\
847,5 \\
\text { nd }\end{array}$ & $\begin{array}{l}\text { nd } \\
\text { nd } \\
\text { nd }\end{array}$ & $\begin{array}{c}19,3 \\
\text { nd } \\
\text { nd }\end{array}$ & $\begin{array}{c}\text { nd } \\
35,4 \\
\text { nd }\end{array}$ & $\begin{array}{c}32,9 \\
2,9 \\
\text { nd }\end{array}$ & $\begin{array}{l}\text { nd } \\
2,9 \\
\text { nd }\end{array}$ & $\begin{array}{c}3,3 \\
184,1 \\
\text { nd }\end{array}$ \\
\hline Alacloro & $\begin{array}{l}10 / 2006 \\
12 / 2006 \\
10 / 2007 \\
\end{array}$ & $\begin{array}{l}\text { nd } \\
\text { nd } \\
\text { nd }\end{array}$ & $\begin{array}{l}\text { nd } \\
\text { nd } \\
\text { nd }\end{array}$ & $\begin{array}{c}11,2 \\
\text { nd } \\
\text { nd } \\
\end{array}$ & $\begin{array}{l}\text { nd } \\
\text { nd } \\
\text { nd }\end{array}$ & $\begin{array}{l}\text { nd } \\
\text { nd } \\
\text { nd }\end{array}$ & $\begin{array}{l}\text { nd } \\
\text { nd } \\
\text { nd }\end{array}$ & $\begin{array}{l}\text { nd } \\
\text { nd } \\
\text { nd }\end{array}$ & $\begin{array}{l}\text { nd } \\
\text { nd } \\
\text { nd }\end{array}$ & $\begin{array}{c}\text { nd } \\
10,9 \\
\text { nd } \\
\end{array}$ \\
\hline Atrazina & $\begin{array}{l}10 / 2006 \\
12 / 2006 \\
10 / 2007\end{array}$ & $\begin{array}{l}2,6 \\
6,0 \\
\text { nd }\end{array}$ & $\begin{array}{l}\text { nd } \\
\text { nd } \\
\text { nd }\end{array}$ & $\begin{array}{l}3,0 \\
3,5 \\
\text { nd }\end{array}$ & $\begin{array}{l}8,5 \\
\text { nd } \\
\text { nd }\end{array}$ & $\begin{array}{c}17,1 \\
252,8 \\
\text { nd }\end{array}$ & $\begin{array}{l}\text { nd } \\
6,0 \\
\text { nd }\end{array}$ & $\begin{array}{c}2,1 \\
2,9 \\
\text { nd }\end{array}$ & $\begin{array}{l}\text { nd } \\
11,0 \\
\text { nd }\end{array}$ & $\begin{array}{c}12,3 \\
6,5 \\
\text { nd }\end{array}$ \\
\hline Diurom & $\begin{array}{l}10 / 2006 \\
12 / 2006 \\
10 / 2007\end{array}$ & $\begin{array}{c}\text { nd } \\
17,4 \\
\text { nd }\end{array}$ & $\begin{array}{l}3,7 \\
\text { nd } \\
\text { nd }\end{array}$ & $\begin{array}{l}4,0 \\
\text { nd } \\
\text { nd }\end{array}$ & $\begin{array}{c}9,5 \\
17,2 \\
\text { nd }\end{array}$ & $\begin{array}{c}14,4 \\
7,8 \\
3,1 \\
\end{array}$ & $\begin{array}{l}\text { nd } \\
3,3 \\
\text { nd }\end{array}$ & $\begin{array}{l}\text { nd } \\
\text { nd } \\
\text { nd }\end{array}$ & $\begin{array}{c}\text { nd } \\
12,4 \\
\text { nd }\end{array}$ & $\begin{array}{c}15,6 \\
\text { nd } \\
\text { nd }\end{array}$ \\
\hline Azoxistrobina & $\begin{array}{l}10 / 2006 \\
12 / 2006 \\
10 / 2007\end{array}$ & $\begin{array}{c}\mathrm{nd} \\
33,4 \\
3,9\end{array}$ & $\begin{array}{l}\text { nd } \\
3,2 \\
\text { nd }\end{array}$ & $\begin{array}{c}12,3 \\
\text { nd } \\
\text { nd }\end{array}$ & $\begin{array}{c}25,8 \\
\text { nd } \\
\text { nd }\end{array}$ & $\begin{array}{c}3,7 \\
\text { nd } \\
46,3\end{array}$ & $\begin{array}{l}\text { nd } \\
\text { nd } \\
\text { nd }\end{array}$ & $\begin{array}{l}\text { nd } \\
\text { nd } \\
\text { nd }\end{array}$ & $\begin{array}{c}\text { nd } \\
42,8 \\
\text { nd }\end{array}$ & $\begin{array}{c}46,8 \\
\text { nd } \\
\text { nd }\end{array}$ \\
\hline Metconazol & $\begin{array}{l}10 / 2006 \\
12 / 2006 \\
10 / 2007\end{array}$ & $\begin{array}{c}\text { nd } \\
152,5 \\
21,8\end{array}$ & $\begin{array}{c}21,7 \\
\text { nd } \\
22,7\end{array}$ & $\begin{array}{c}\text { nd } \\
\text { nd } \\
53,3\end{array}$ & $\begin{array}{c}\text { nd } \\
\text { nd } \\
24,4\end{array}$ & $\begin{array}{c}\text { nd } \\
\text { nd } \\
52,2\end{array}$ & $\begin{array}{c}\text { nd } \\
\text { nd } \\
13,9\end{array}$ & $\begin{array}{c}\text { nd } \\
\text { nd } \\
55,0\end{array}$ & $\begin{array}{l}\text { nd } \\
\text { nd } \\
\text { nd }\end{array}$ & $\begin{array}{l}\text { nd } \\
\text { nd } \\
\text { nd }\end{array}$ \\
\hline Metalaxil & $\begin{array}{l}10 / 2006 \\
12 / 2006 \\
10 / 2007\end{array}$ & $\begin{array}{c}15,6 \\
40,6 \\
\text { nd } \\
\end{array}$ & $\begin{array}{c}\mathrm{nd} \\
41,0 \\
\mathrm{nd}\end{array}$ & $\begin{array}{l}9,6 \\
5,0 \\
\text { nd } \\
\end{array}$ & $\begin{array}{c}26,6 \\
252,8 \\
3,6 \\
\end{array}$ & $\begin{array}{c}80,4 \\
\text { nd } \\
\text { nd }\end{array}$ & $\begin{array}{c}7,4 \\
49,7 \\
\text { nd } \\
\end{array}$ & $\begin{array}{c}17,9 \\
29,7 \\
\text { nd } \\
\end{array}$ & $\begin{array}{c}11,4 \\
36,3 \\
\text { nd }\end{array}$ & $\begin{array}{c}\text { nd } \\
30,0 \\
\text { nd }\end{array}$ \\
\hline Tebuconazol & $\begin{array}{l}10 / 2006 \\
12 / 2006 \\
10 / 2007\end{array}$ & $\begin{array}{c}34,8 \\
66,7 \\
\text { nd } \\
\end{array}$ & $\begin{array}{c}11,4 \\
64,1 \\
\text { nd } \\
\end{array}$ & $\begin{array}{c}13,3 \\
16,1 \\
4,8 \\
\end{array}$ & $\begin{array}{c}38,8 \\
\text { nd } \\
\text { nd }\end{array}$ & $\begin{array}{l}\text { nd } \\
\text { nd } \\
\text { nd }\end{array}$ & $\begin{array}{c}\text { nd } \\
52,9 \\
\text { nd }\end{array}$ & $\begin{array}{c}38,5 \\
69,5 \\
\text { nd }\end{array}$ & $\begin{array}{c}\text { nd } \\
\text { nd } \\
129,9\end{array}$ & $\begin{array}{c}52,1 \\
\text { nd } \\
334,5\end{array}$ \\
\hline Lambda-cialotrina & $\begin{array}{l}10 / 2006 \\
12 / 2006 \\
10 / 2007\end{array}$ & $\begin{array}{c}\text { nd } \\
4,3 \\
12,4\end{array}$ & $\begin{array}{c}4,6 \\
\text { nd } \\
10,7\end{array}$ & $\begin{array}{l}2,8 \\
6,7 \\
\text { nd }\end{array}$ & $\begin{array}{c}64,3 \\
\text { nd } \\
2,18\end{array}$ & $\begin{array}{c}43,6 \\
\text { nd } \\
13,9\end{array}$ & $\begin{array}{l}18,3 \\
\text { nd } \\
\text { nd }\end{array}$ & $\begin{array}{l}4,6 \\
\text { nd } \\
\text { nd }\end{array}$ & $\begin{array}{c}\text { nd } \\
\text { nd } \\
12,5\end{array}$ & $\begin{array}{l}3,1 \\
8,4 \\
4,7\end{array}$ \\
\hline
\end{tabular}


O efeito dos macroporos sobre o transporte químico está relacionado não apenas ao transporte advectivo mas também ao tempo de residência na zona biologicamente ativa do solo. Estudos têm mostrado que, devido aos caminhos preferenciais, o tempo de avanço dos pesticidas é independente de suas propriedades adsortivas. Isto indica que são necessários estudos no campo para verificar os parâmetros de transporte e adsorção para pesticidas (Malone et al., 2004; Kazemi et al., 2009). Os pesticidas submetidos aos processos de degradação e sorção as partículas do solo, são transportados na direção vertical pela água percolada e as quantidades transportadas serão mais expressivas se a percolação ocorrer em caminhos preferenciais formados pelos macroporos; neste contexto o efeito de retardamento provocado pela adsorção aos colóides do solo, seria reduzido; os macroporos ocorrem de forma irregular, a coleta aleatória de amostras no perfil do solo, seja ela simples ou composta, não coincide necessariamente em todos os eventos, com a ocorrência de macroporos, cujo resultado prevê que as quantidades de pesticidas encontrados na amostra de solo coletado sejam reduzidas ou mesmo nulas.

\section{CONCLUSÕES}

1. Os pesticidas estudados foram detectados ao longo do perfil do solo, com frequência variando entre 0 e 77,8\%, e os fungicidas apresentando maior frequência de detecção; no geral, os pesticidas tiveram maior frequência na camada mais superficial do solo.

2. Os valores das concentrações variaram muito, tanto na amostragem simples quanto na composta, demonstrando que o procedimento amostral não deve ter influenciado nos resultados.

3. Não houve continuidade das concentrações, seja nas áreas quanto nas profundidades.

4. Os herbicidas, fungicidas e inseticidas estudados tiveram mobilidade no perfil do solo, tendo-se encontrado resíduos na camada de 80 a 100 cm; não se detectou correlação entre as concentrações dos ingredientes ativos e o índice GUS.

\section{Agradecimentos}

Os autores agradecem ao CNPq (Proc 301156/2008-5), ao FUNAPES-FURB e à FINEP pelo suporte financeiro.

\section{LITERATURA CITADA}

AGRITOX - Base de données sur les substances actives phytopharmaceutiques. http://www.dive.afssa.fr/agritox/ guides/guide-agritox.html. 10 Fev. 2009.

Al-Rajab, A. J.; Amellal, S.; Schiavon, M. Sorption and leaching of C-14-glyphosate in agricultural soils. Agronomy for Sustainable Development, v.28, p.419-428, 2008.
Bergstrom, L. F. Symposium 2 part 2: Food production for a growing world population - The impact of food production on soils and groundwater resources. Journal of Food Science, v.69, p.R181-R184, 2004.

Blanchard, P. E.; Lerch, R. N. Watershed vulnerability to losses of agricultural chemicals: Interactions of chemistry, hydrology, and land-use. Environmental Science \& Technology, v.34, p.3315-3322, 2000.

Cantarutti, T. F. P.; Araújo, S. L. D.; Rossi, S. C.; Dalsenter, P. R. Resíduos de pesticidas em alimentos. Revista de Ecotoxicologia e Meio Ambiente., v.18, p.9-16, 2009.

Correia, F. V.; Langenbach, T. Dinâmica da distribuição e degradação de atrazina em argissolo vermelho-amarelo sob condições de clima tropical úmido. Revista Brasileira de Ciência do Solo, v.30, p.183-192, 2006.

Delgado-Moreno, L.; Sanchez, L.; Castillo, A.; Pot, V.; Pena, A. Behavior of bensulfuron-methyl in an agricultural alkaline soil. Journal of Environmental Science and Health, Part BPesticides Food Contaminants and Agricultural Wastes, v.42, p.241-248, 2007.

EMBRAPA - Empresa Brasileira de Pesquisa Agropecuária. Centro Nacional de Pesquisa de Solos, Sistema brasileiro de classificação de solos. Rio de Janeiro: EMBRAPA, 1999. 412p.

Gibson, G.; Koifman, S. Agricultural toxic use and temporal distribution of male birth rate in the state of Parana, Brazil. Revista Panamericana de Salud Publica-Pan American Journal of Public Health, v.24, p.240-247, 2008.

Gustafson, D. I. Groundwater Ubiquity Score - a Simple method for assessing pesticide leachability. Environmental Toxicology and Chemistry, v.8, p.339-357, 1989.

Hartemink, A. E. Sugarcane for bioethanol: Soil and environmental issues. Advances in Agronomy, v.99, p.125182, 2008.

Johnson, R. M.; Sims, J. T. Influence of surface and subsoil properties on herbicide sorption by atlantic coastal-plain soils. Soil Science, v.155, p.339-348, 1993.

Kaur, R.; Kumar, S.; Gurung, H. P. A pedo-transfer function (PTF) for estimating soil bulk density from basic soil data and its comparison with existing PTFs. Australian Journal of Soil Research, v.40, p.847-857, 2002.

Kazemi, H. V.; Anderson, S. H.; Goyne, K. W.; Gantzer, C. J. Aldicarb and carbofuran transport in a Hapludalf influenced by differential antecedent soil water content and irrigation delay. Chemosphere, v.74, p.265-273, 2009.

Kromer, T.; Ophoff, H.; Stork, A.; Fuhr, F. Photodegradation and volatility of pesticides - Chamber experiments. Environmental Science and Pollution Research, v.11, p.107120, 2004.

Lapworth, D. J.; Gooddy, D. C.; Stuart, M. E.; Chilton, P. J.; Cachandt, G.; Knapp, M.; Bishop, S. Pesticides in groundwater: Some observations on temporal and spatial trends. Water and Environment Journal, v.20, p.55-64, 2006.

Malone, R. W.; Shipitalo, M. J.; Meek, D. W. Relationship between herbicide concentration in percolate, percolate breakthrough time, and number of active macropores. Transactions of the ASAE, v.47, p.1453-1456, 2004. 
Matallo, M. B.; Spadotto, C. A.; Luchini, L. C.; Gomes, M. A. F. Sorption, degradation, and leaching of tebuthiuron and diuron in soil columns. Journal of Environmental Science and Health, Part B-Pesticides Food Contaminants and Agricultural Wastes, v.40, p.39-43, 2005.

Molozzi, J.; Pinheiro, A.; Silva, M. R. Qualidade da água em diferentes estádios de desenvolvimento do arroz irrigado. Pesquisa Agropecuaria Brasileira, v.41, p.1393-1398, 2006.

Papiernik, S. K.; Koskinen, W. C.; Cox, L.; Rice, P. J.; Clay, S. A.; Werdin-Pfisterer, N. R.; Norberg, K. A. Sorption-desorption of imidacloprid and its metabolites in soil and vadose zone materials. Journal of Agricultural and Food Chemistry, v.54, p.8163-8170, 2006.

Peres, F.; Moreira, J. C. Health, environment, and pesticide use in a farming area in Rio de Janeiro State, Brazil. Cadernos de Saúde Pública, v.23, p.S612-S621, 2007.

Ramos, H. H.; Maziero, J. V. G.; Yanai, K.; Corrêa, I. M.; Severino, F. J.; Kanno, O. Y.; Martins, P. S.; Mura, C.; Morgano, M. A. Exposição dérmica do aplicador de agrotóxicos na cultura da uva, com diferentes pulverizadores. Revista Brasileira de Engenharia Agrícola e Ambiental, v.6, p.175-179, 2002.

Ruggieri, F.; D’archivio, A. A.; Fanelli, M.; Mazzeo, P.; Paoletti, E. A multi-lysimeter investigation on the mobility and persistence of pesticides in the loam soil of the Fucino Plain (Italy). Journal of Environmental Monitoring, v.10, p.747-752, 2008.

Sakaliene, O.; Papiernik, S. K.; Koskinen, W. C.; Spokas, K. A. Sorption and predicted mobility of herbicides in Baltic soils. Journal of Environmental Science and Health Part BPesticides Food Contaminants and Agricultural Wastes, v.42, p.641-647, 2007.

Schaffrath, V. R.; Tormena, C. A.; Fidalski, J.; Goncalves, A. C. A. Variability and correlation among physical properties of an Oxisol under no-till and conventional tillage. Revista Brasileira de Ciência do Solo, v. 32,p.1369-1377, 2008.
SINDAG - Sindicato Nacional da Indústria de Produtos para Defesa Agrícola. http://www.sindag.com.br/. 05 Fev.2009.

Siqueira, S. L.; Kruse, M. H. L. Agrochemicals and human health: contributions of healthcare professionals. Revista da Escola de Enfermagem da USP, v.42, p.573-579, 2008.

Vasconcelos, F. C. W.; Nóbrega, J. C. A.; Guerreiro, M. C.; Jesus, E. A.; Julião, L. G. F.; Curi, N. Mobilidade do herbicida atrazina em amostras de latossolos vermelhos sob calagem e adubação fosfatada. Pesticidas: Revista de Ecotoxicologia e Meio Ambiente, v.18, p.133-146, 2009.

Vieira, E.O.; Prates, H.T.; Pereira, J.R.B.; Silva,G.F.; Duarte, F.V.; Neres, P. M. Avaliação da contaminação do carbofuran nos solos do Distrito de Irrigação do Gorutuba. Revista Brasileira de Engenharia Agrícola e Ambiental, v.13, p.250-256, 2009.

Wade, H. F.; York, A. C.; Morey, A. E.; Padmore, J. M.; Rudo, K. M. The impact of pesticide use on groundwater in North Carolina. Journal of Environmental Quality, v.27, p.10181026, 1998.

Waichman, A. V.; Eve, E.; Nina, N. C. D. Do farmers understand the information displayed on pesticide product labels? A key question to reduce pesticides exposure and risk of poisoning in the Brazilian Amazon. Crop Protection, v.26, p.576-583, 2007.

Wu, L.; Liu, G.; Yates, M. V.; Green, R. L.; Pacheco, P.; Gan, J.; YateS, S. R. Environmental fate of metalaxyl and chlorothalonil applied to a bentgrass putting green under southern California climatic conditions. Pest Management Science, v.58, p.335-342, 2002.

Yao, Y.; Harner, T.; Blanchard, P.; Tuduri, L.; Waite, D.; Poissant, L.; Murphy, C.; Belzer, W.; Aulagnier, F.; Sverko, E. Pesticides in the atmosphere across Canadian agricultural regions. Environmental Science \& Technology, v.42, p.5931-5937, 2008. 